\title{
Evaluation des inefficiences zootechnique et environnementale pour intensifier écologiquement les systèmes d'élevage tropicaux. Etude de cas à la Réunion
}

\begin{abstract}
Mots-clés
Production animale - Analyse du cycle de vie - Intensification - Durabilité Impact sur l'environnement - Réunion.
\end{abstract}

\author{
J. Vayssières ${ }^{1 *}$ A. Thevenot ${ }^{1} \mathrm{M}$. Vigne ${ }^{1} \mathrm{M}_{\text {. Cano }}{ }^{1}$ \\ A. Broc ${ }^{1}$ R. Bellino ${ }^{1}$ E. Diacono ${ }^{1}$ B. de Laburthe ${ }^{2}$ \\ J.L. Bochu ${ }^{3}$ E. Tillard ${ }^{1}$ P. Lecomte ${ }^{1}$
}

\section{Résumé}

Selon la FAO, I'élevage contribuerait à hauteur de 18 p. 100 aux émissions globales de gaz à effet de serre (GES) d'origine anthropique. Face à une population mondiale et une demande en produits animaux grandissantes, il s'agit de concevoir des systèmes d'élevage non seulement plus productifs mais également plus respectueux de l'environnement. Dans cette perspective, les consommations d'énergies non renouvelables (ENR) et les émissions de GES des principales productions animales de la Réunion (bovin lait, bovin viande, porc, volaille et lapin) ont été évaluées. Partant d'une méthode développée en France métropolitaine, il s'agissait de réévaluer les coefficients énergétiques et les facteurs d'émission en tenant compte des particularités du contexte et des systèmes d'élevage locaux. L'échantillon étudié comprenait 195 élevages, soit plus de 25 p. 100 des exploitations encadrées par les coopératives locales. Cette étude a montré que les inefficiences environnementales (consommations d'ENR et émissions de GES par kilogramme de produit animal) et l'inefficience zootechnique (quantités d'aliments concentrés consommés par kilogramme de produit animal) étaient corrélées positivement. Il est donc possible d'intensifier écologiquement les productions animales. De telles études sont rares dans les Suds ; leur essor suppose des adaptations méthodologiques encore plus importantes que celles menées dans le cas réunionnais, pour pouvoir évaluer des systèmes généralement peu mécanisés, à faible niveau d'intrants, mixtes, dont l'élevage est multifonctionnel et mobilise des formes d'énergies multiples.

\section{INTRODUCTION}

Le réchauffement climatique est maintenant avéré et inéluctable (11). Les activités anthropiques ont un rôle essentiel dans le réchauffement climatique de notre planète. Il résulte principalement d'un accroissement rapide de la concentration en GES de

1. Cirad, UMR Systèmes d'élevage méditerranéens et tropicaux, station de Ligne Paradis, 7 ch. de l'IRAT, St-Pierre, la Réunion.

2. FRCA, St-Pierre, la Réunion.

3. Solagro, Toulouse, France.

* Auteur pour la correspondance

Tél. : +221775418362; fax : +221338211879

E-mail : jonathan.vayssieres@cirad.fr l'atmosphère. Cet accroissement est lui-même fortement lié aux consommations d'ENR en plein essor depuis l'industrialisation dans les années 1950 des pays développés, puis encore accentué par celle des pays émergents ces dix dernières années.

La place des activités d'élevage n'est pas négligeable puisqu'il est estimé qu'elles sont à l'origine, directement ou indirectement, de 18 p. 100 des émissions de GES (17). Cette estimation comptabilise : - les émissions indirectes résultant de la production, du transport des intrants et des produits des activités d'élevage, et de la mise en culture ou en prairies d'espaces naturels et de forêts pour cette production (déstockage du carbone du sol et de la végétation ligneuse) ;

- les émissions directes liées à la fermentation entérique des ruminants, la gestion des effluents d'élevage et la fertilisation 
organique et minérale des cultures et prairies destinées à l'alimentation des animaux.

Pour la recherche zootechnique il s'agit non seulement d'anticiper les conséquences (positives ou négatives) que le réchauffement climatique pourrait avoir sur les systèmes d'élevage pour faciliter leur adaptation (20), mais aussi d'accompagner une mitigation des émissions de GES liées aux activités d'élevage. Ce processus est déjà en réflexion et parfois amorcé dans les pays industrialisés signataires du protocole de Kyoto. Ces derniers se sont en effet engagés à réduire de 20 p. 100 leurs émissions de GES d'ici 2050. Les pays émergents en développement et signataires n'ont pas pour l'instant de contraintes spécifiques. C'est cependant dans ces pays que la croissance des émissions risque d'être la plus importante dans les quarante prochaines années. En effet, la population est en forte croissance et ses comportements de consommation sont en pleine mutation. La prospective Agrimonde prévoit que le continent africain en particulier génère une part majeure de la croissance démographique mondiale à venir (6). Cette croissance devrait s'accompagner d'une rapide augmentation de la demande en produits agricoles, et tout particulièrement en produits animaux, si le changement des habitudes alimentaires suit celui déjà intervenu en pays industrialisés et en cours en pays émergents. Le développement de méthodes permettant l'évaluation des consommations d'ENR et des émissions de GES générées par les activités d'élevage dans les pays du Sud est une étape importante pour amorcer leur mitigation et l'accroissement de leur efficience multicritères $(22)$.

\section{MATERIEL ET METHODES}

\section{Méthode originelle et indicateurs d'inefficience calculés}

La méthode Planète, retenue dans cette étude, reprend les grands principes de l'analyse de cycle de vie $(\mathrm{ACV})$ en se limitant à deux des impacts classiquement évalués : les consommations d'ENR et les émissions de GES (15). Autre particularité, son périmètre d'analyse s'arrête aux portes de la ferme et ne considère pas la commercialisation du produit et le recyclage de ses coproduits. Cette méthode est donc incomplète pour comparer des produits mais particulièrement adaptée pour comparer différents systèmes de production.

Elle permet de calculer deux indicateurs d'inefficience environnementale : les consommations totales d'ENR et les émissions totales de GES dans les deux cas ramenées à l'unité de produit animal. L'indicateur d'inefficience zootechnique est l'indice de consommation des aliments, c'est-à-dire la quantité totale d'aliments concentrés consommés divisée par la quantité totale de produits animaux générés par l'exploitation. L'unité de produit diffère selon la production animale considérée. Elle correspond au kilogramme de lait pour la production bovine laitière et au kilogramme de viande pour les autres productions.

\section{Adaptation de la méthode}

Les paramètres proposés dans l'outil Planète sont uniquement valables pour évaluer des productions agricoles en France métropolitaine (3). Un important travail d'adaptation des coefficients énergétiques et des facteurs d'émission a donc été entrepris (19). Pour les coefficients énergétiques, ce travail a été effectué principalement selon le trois modalités suivantes :

- lorsque les intrants étaient de même nature et importés de France (cas du matériel agricole, par exemple), le coefficient initial a été conservé et un coût énergétique a été ajouté pour le transport depuis la France métropolitaine jusqu'à la Réunion (soit $10600 \mathrm{~km}$ en fret maritime);

- si les intrants étaient de même nature mais avaient des origines différentes, le coût énergétique du transport initial a été substitué par un coût nouvellement calculé. Par exemple, le fioul consommé à la Réunion avait une origine différente. Les coûts énergétiques attribués pour l'extraction et le raffinage ont été conservés mais le transport entre Mer du Nord, Proche Orient et France métropolitaine a été remplacé par un transport entre Singapour et la Réunion ;

- certains intrants étaient particuliers aux systèmes de production réunionnais. Dans ce cas, un nouveau coefficient énergétique a été calculé. C'est le cas, par exemple, du coefficient énergétique de l'ensilage produit à la Réunion qui a été calculé selon l'itinéraire technique moyen et les différents intrants consommés lors des différentes étapes du cycle de production (création de la prairie, entretien, récolte de l'ensilage et transport sur l'exploitation).

Concernant les facteurs d'émission de GES, la démarche d'adaptation est très similaire à celle des coefficients énergétiques à l'exception des émissions de GES directes (essentiellement des émissions de $\mathrm{CH}_{4}$ d'origine entérique et de $\mathrm{N}_{2} \mathrm{O}$ issues de la gestion des effluents d'élevage). Dans ce cas, le manque de résultats expérimentaux locaux nous a contraint de reprendre de grands standards internationaux proposés par l'Intergovernmental Panel on Climate Change (11), alors que ces coefficients d'émission mériteraient d'être précisés du fait de la particularité des conditions climatiques et des pratiques d'élevage locales (par exemple, rations relativement pauvres en cellulose pour les ruminants).

\section{Recueil des données de terrain}

Cette étude a couvert les principales productions animales de l'île : l'élevage de bovin lait, de bovin viande, de porc, de volaille et de lapin. L'échantillon étudié a comporté 195 élevages, soit plus de 25 p. 100 des exploitations encadrées par les coopératives locales. Ces dernières ont participé à l'échantillonnage des élevages. L'échantillonnage a été réalisé à dires d'experts avec l'objectif de couvrir la diversité des systèmes d'élevage rencontrés sur l'île selon des critères de performances techniques, de taille et de localisation.

Nous avons pu bénéficier des comptabilités des éleveurs grâce à l'appui de leurs centres de gestion. Ces comptabilités ont été précieuses pour quantifier les intrants et les produits de l'exploitation. Les données ont pu être croisées avec les données individuelles disponibles auprès des entreprises d'approvisionnement en intrants (par exemple, fournisseurs d'aliments) et des coopératives se chargeant de l'écoulement des produits.

\section{RESULTATS}

Les tableaux I et II montrent respectivement les consommations d'ENR et les émissions de GES totales ramenées à l'unité de produit. Ces indicateurs sont des moyennes par production pour l'année 2007. Le coefficient de variation associé à ces moyennes, les deux principaux postes de consommation et d'émission sont également donnés. Le coût de l'insularité correspond au pourcentage de la consommation d'ENR (tableau I) et des émissions de GES (tableau II) totales imputables au transport des intrants (depuis la France métropolitaine et l'Amérique du Sud principalement).

\section{Variabilité interproductions et intraproductions}

Cette étude montre une forte disparité des résultats entre productions animales. L'élevage de bovin lait a été difficilement 


\section{Tableau I}

Consommation d'énergie non renouvelable et principaux postes de consommation par production animale à la Réunion (2007)

\begin{tabular}{|c|c|c|c|c|c|c|c|}
\hline \multirow[t]{2}{*}{ Production } & \multicolumn{2}{|c|}{$\begin{array}{l}\text { Consommation } \\
\text { énergétique totale }\end{array}$} & \multicolumn{4}{|c|}{$\begin{array}{l}\text { Principaux postes } \\
\text { de consommation }\end{array}$} & \multirow{2}{*}{$\begin{array}{c}\begin{array}{c}\text { Coût de } \\
\text { l'insularité }\end{array} \\
\%{ }^{3}\end{array}$} \\
\hline & $\begin{array}{c}\text { Moyenne } \\
\text { 1J / unité de produit) } 1\end{array}$ & $\begin{array}{c}\text { Coefficient } \\
\text { de variation }(\%)\end{array}$ & $1^{\mathrm{er}}$ poste & $\%^{2}$ & $2^{\mathrm{e}}$ poste & $\%^{2}$ & \\
\hline Bovin lait $(n=30)$ & 7 & 17 & Alimentation & 62 & Carburant & 12 & 29 \\
\hline Bovin viande naisseur $(\mathrm{n}=19)$ & 59 & 33 & Alimentation & 31 & Carburant & 23 & 17 \\
\hline Bovin viande engraisseur $(n=15)$ & 62 & 28 & Alimentation & 53 & Carburant & 16 & 25 \\
\hline Porc $(n=36)$ & 23 & 19 & Alimentation & 77 & Electricité et gaz & 10 & 38 \\
\hline Volaille $(\mathrm{n}=70)$ & 22 & 19 & Alimentation & 75 & Electricité et gaz & 13 & 32 \\
\hline Lapin $(\mathrm{n}=25)$ & 47 & 33 & Alimentation & 59 & Electricité et gaz & 14 & 23 \\
\hline
\end{tabular}

${ }^{1}$ L'unité de produit est le kg de lait brut pour l'élevage de bovin lait. Pour le reste des productions il s'agit du kg de viande vif.

2 Pourcentage de la consommation énergétique totale.

${ }^{3}$ Pourcentage de la consommation énergétique totale imputable au transport des intrants depuis la France métropolitaine et l'Amérique du Sud.

\section{Tableau II}

Emissions de gaz à effet de serre et principaux postes d'émission par production animale à la Réunion (2007)

\begin{tabular}{|c|c|c|c|c|c|c|c|}
\hline \multirow[t]{2}{*}{ Production } & \multicolumn{2}{|c|}{ Emissions de GES * totales } & \multicolumn{4}{|c|}{ Principaux postes d'émission } & \multirow{2}{*}{$\begin{array}{c}\begin{array}{c}\text { Coût de } \\
\text { l'insularité }\end{array} \\
\%{ }^{3}\end{array}$} \\
\hline & $\begin{array}{l}\text { Moyenne } \\
\text { eq / unité de produit) }{ }^{1} \text { de }\end{array}$ & $\begin{array}{l}\text { Coefficient } \\
\text { e variation }(\%)\end{array}$ & $1^{\mathrm{er}}$ poste & $\%^{2}$ & $2^{\mathrm{e}}$ poste & $\% 2$ & \\
\hline Bovin lait $(n=30)$ & 1,8 & 22 & Alimentation & 40 & $\begin{array}{l}\text { Fermentation } \\
\text { entérique }\end{array}$ & 27 & 20 \\
\hline Bovin viande naisseur $(\mathrm{n}=19)$ & 18,5 & 30 & $\begin{array}{l}\text { Fermentation } \\
\text { entérique }\end{array}$ & 55 & Alimentation & 15 & 9 \\
\hline Bovin viande engraisseur $(n=15)$ & 10,4 & 24 & Alimentation & 44 & $\begin{array}{l}\text { Fermentation } \\
\text { entérique }\end{array}$ & 30 & 24 \\
\hline Porc $(n=36)$ & 3,6 & 14 & Alimentation & 75 & Gestion effluents & 9 & 39 \\
\hline Volaille $(n=70)$ & 2,8 & 16 & Alimentation & 80 & Gestion effluents & 8 & 42 \\
\hline Lapin $(\mathrm{n}=25)$ & 5,2 & 25 & Alimentation & 65 & Electricité et gaz & 9 & 34 \\
\hline
\end{tabular}

comparable étant donné son unité de produit différente. Pour les élevages ayant la viande comme produit principal, les monogastriques (à l'exception des lapins) ont eu physiologiquement une meilleure efficience d'utilisation des aliments que les ruminants et ont produit peu d'émissions de GES d'origine entérique, ce qui expliquait leurs consommations d'ENR et leurs émissions de GES plus faibles. Les élevages de lapins étant particulièrement affectés par des problèmes sanitaires (forte mortalité avant sevrage), leurs consommations et émissions se rapprochaient des consommations des élevages de ruminants. Pour les émissions de GES, ces différences entre ruminants et monogastriques auraient été moindres si le stockage du carbone par les prairies avait été pris en compte, ce qui n'a pas été le cas dans cette étude.

Au-delà de ces comparaisons interproductions, de fortes variations intraproduction des consommations d'ENR et des émissions de
GES ont surtout été relevées, puisque les coefficients de variation ont tous été supérieurs à 14 p. 100 . Cette variabilité a été particulièrement élevée pour les élevages de bovins viande naisseurs et engraisseurs, et pour les élevages de lapins. Une moindre variabilité $(\mathrm{CV}<20$ p. 100) a été observée pour les élevages de porcs et de volailles, car les pratiques y étaient plus standardisées.

\section{Principaux postes d'impact}

L'alimentation a été le principal poste de consommation et d'émission. Il totalisait les consommations et les émissions intervenues pendant la production des matières premières, leur transformation, leur conditionnement et leur transport. Selon la production, ce poste représentait 31 à 77 p. 100 des consommations énergétiques totales et 15 à 80 p. 100 des émissions de GES. La contribution 
de l'alimentation a été particulièrement élevée car les élevages (y compris ceux de ruminants) avaient fortement recours aux aliments concentrés dont la matière première était importée d'Europe (céréales) et d'Amérique du Sud (soja). Le deuxième poste énergétique a été généralement le carburant pour les ruminants et l'électricité pour les monogastriques. En effet, les élevages de ruminants exploitaient des surfaces fourragères importantes et les monogastriques étaient élevés en bâtiments.

Le deuxième poste d'émission résultait de la fermentation entérique pour les ruminants et de la gestion des effluents d'élevage pour les monogastriques. Enfin, mis à part l'élevage de bovins viande naisseurs qui valorisait des surfaces prairiales importantes, le coût de l'insularité des productions animales réunionnaises a été particulièrement élevé aussi bien en termes de consommation énergétique ( $\geq 23$ p. 100) que d'émission de GES ( $\geq 20$ p. 100). Ce surcoût reflétait leur forte dépendance vis-à-vis de l'Europe et de l'Amérique du Sud, les territoires d'origine de la majorité des intrants, et il a été accentué par les niveaux élevés de concentrés distribués aux ruminants.

\section{Corrélations entre inefficiences zootechnique et environnementales}

Les figures 1 et 2 représentent respectivement les consommations énergétiques et les émissions totales de GES par kilogramme de poids vif produit (inefficiences environnementales) en fonction de l'indice de consommation des aliments concentrés (inefficience zootechnique). Seules les productions présentant les corrélations les plus élevées ont été représentées. L'élevage bovin laitier n'y figure pas car la différence d'unité de produit implique un écart d'échelle trop important pour être représenté sur une même figure. Les droites de régression linéaire entre les variables environnementales et la variable technique sont représentées dans les figures 1 et 2 . En référence aux $\mathrm{R}^{2}$, les résultats montrent une corrélation positive entre les inefficiences environnementales et l'inefficience zootechnique, en cohérence avec le fait que l'alimentation représentait le principal poste de consommation et d'émission (tableaux I et II). La corrélation a été particulièrement élevée pour la production de volaille où les $\mathrm{R}^{2}$ étaient dans les deux cas supérieurs à 0,9 .

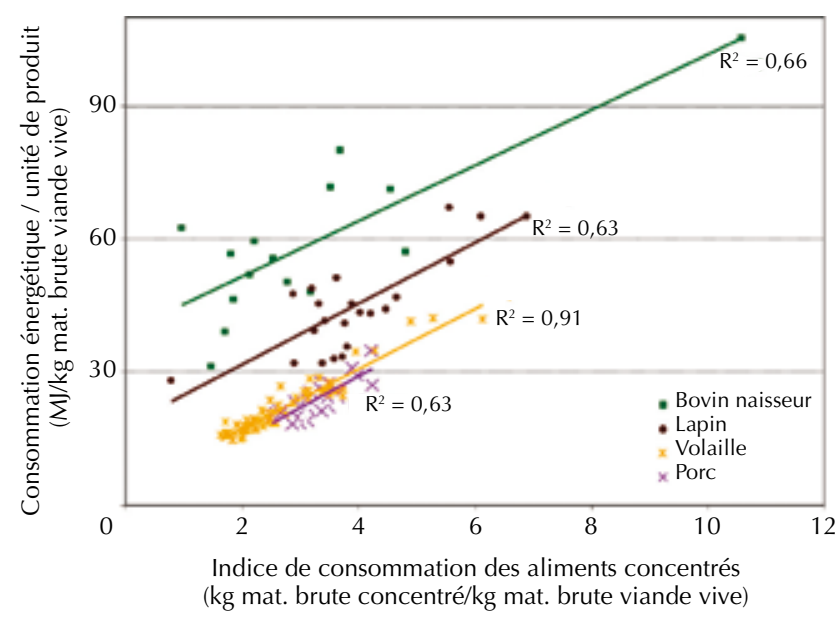

Figure 1 : relation entre les consommations d'énergie non renouvelable et la consommation d'aliments concentrés par kilogramme de poids vif produits dans différentes exploitations en comparant des systèmes d'élevage bovins naisseurs, lapins, poulets de chair et porcins à la Réunion (2007).
Elle n'a pas été vérifiée pour l'émission de GES des productions de ruminants du fait de la forte contribution des fermentations entériques (27-55 p. 100 des émissions de GES) et des émissions de $\mathrm{N}_{2} \mathrm{O}$ pendant le stockage et les manipulations des effluents d'élevage. En effet, ces deux sources d'émission ont été essentiellement liées au nombre d'animaux et non pas aux quantités d'aliments concentrés consommées.

\section{Voies de mitigation}

Au-delà de la simple quantification de l'impact probable des productions animales, l'intérêt d'une telle étude couvrant un nombre relativement important d'exploitations a été de permettre l'identification de voies de mitigation adaptées aux systèmes d'élevage locaux. A la Réunion, la forte variabilité des performances au sein de chaque production animale évoquée plus haut ouvre des perspectives de marges de progrès importantes. Etant donné la multiplicité des microclimats, une étude approfondie de l'effet climat (température, hygrométrie et pluviométrie) sur les inefficiences mériterait d'être menée afin de déterminer, d'une part, si les inefficiences sont plutôt dues aux pratiques agricoles ou à la localisation des élevages et, d'autre part, déterminer si la localisation préférentielle des élevages dans des zones pédoclimatiques favorables ne serait pas une voie de mitigation à envisager.

En référence aux principaux postes de consommation et d'émission (tableaux I et II), des voies de mitigation ont été identifiées. Les surfaces agricoles de l'île sont relativement réduites et leurs possibilités d'expansion limitées (fortes tensions sur le foncier). L'importation de céréales et de tourteaux de soja est donc nécessaire pour garder les niveaux de productions actuels. Privilégier des sources d'approvisionnement proches de la Réunion (par exemple, Mozambique) serait favorable à la mitigation des deux impacts environnementaux étudiés. De plus, quelle que soit l'espèce élevée, une réduction de l'indice de consommation des aliments concentrés doit être visée. Pour les ruminants cela passe essentiellement par une amélioration de la qualité des fourrages distribués (en remplacement d'une partie des concentrés) et un meilleur suivi de la reproduction (réduction de l'intervalle vêlage - vêlage). Pour les monogastriques, la réduction de l'indice de consommation des aliments concentrés passe avant tout par

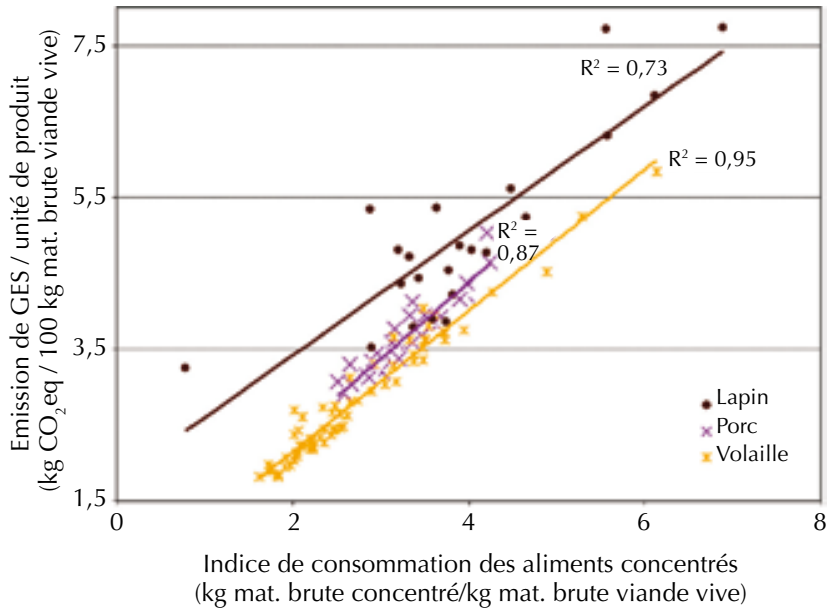

Figure 2 : relation entre les émissions de gaz à effet de serre (GES) et la consommation d'aliments concentrés par kilogramme de poids vif produit dans différentes exploitations en comparant des systèmes d'élevage lapins, porcins et poulets de chair à la Réunion (2007). 
une amélioration de l'ambiance dans les bâtiments (par exemple, réduction de la température en été) et plus de rigueur en matière de biosécurité (hygiène, prévention des pathologies) qui sont deux points clés en contexte tropical.

Pour réduire la consommation énergétique des élevages de bovins en carburant et des monogastriques en électricité et en butane, des progrès importants peuvent également être réalisés, a) par le réglage et le choix de tracteurs de puissances adaptées aux travaux réalisés en élevage de ruminants, b) par l'isolation des bâtiments et la mise en place de boîtiers de régulation d'ambiance automatisés en élevage de monogastriques (coût des boîtiers largement compensé par les économies).

Enfin, une amélioration de la qualité des fourrages produits localement favorisera également une diminution des émissions de $\mathrm{CH}_{4}$ liées aux fermentations entériques (deuxième poste d'émission des ruminants) et une couverture des fosses permettra de réduire les émissions de $\mathrm{N}_{2} \mathrm{O}$ liées au stockage des effluents d'élevage (deuxième poste d'émission des monogastriques).

\section{DISCUSSION}

Les systèmes d'élevage réunionnais différent de ceux rencontrés en France métropolitaine. En particulier en élevage de bovins, les niveaux de consommation de concentrés sont plus élevés à la Réunion. Par ailleurs, les élevages de monogastriques en horssol dépensent sur cette île généralement moins d'énergie pour le chauffage des bâtiments. Dans les deux cas, l'importance énergétique en regard du poste alimentation est donc accentuée. Cette particularité des systèmes d'élevage réunionnais nous a conduit à fortement détailler les coefficients associés au poste alimentation ; un coefficient énergétique a été défini pour chaque type d'aliment consommé en fonction de sa composition et de l'origine de ses composants (plus de 150 aliments distingués), alors que la méthode originelle prévoyait un coefficient moyen par production animale (soit moins d'une dizaine d'aliments distingués). Cette expérience de transposition d'une méthode de type ACV dans un contexte tropical différent de celui de sa conception constitue une première référence dans la perspective d'étudier des systèmes de production agricoles dans les pays du Sud.

Peu de références sont disponibles concernant les systèmes d'élevage tropicaux en matière de consommation d'ENR et d'émission de GES (1). Bien souvent, ces systèmes d'élevage s'éloignent encore plus significativement de ceux rencontrés en France métropolitaine. Généralement, ils sont moins productifs, moins mécanisés et ont peu recours aux intrants industriels importés. Par conséquent, les consommations d'ENR ramenées au kilogramme de produit y sont plus faibles $(2,23)$. De plus les systèmes d'élevage des Suds sont majoritairement alimentés sur la base de ressources locales fortement cellulosiques à haut pouvoir méthanogène (par exemple, résidus de cultures). Ainsi, les premières ACV montrent des émissions de GES par kilogramme de produit plus élevées $(17,9)$.

Une application dans les Suds de méthodes telles que Planète permettrait d'élargir le panel de références et d'identifier des voies de mitigation cohérentes avec les voies d'intensification locales. Cela suppose de fortes adaptations méthodologiques. La principale semble être une adaptation du périmètre d'analyse pour une meilleure prise en compte de la consommation énergétique de la main d'œuvre mobilisée pour conduire les troupeaux et pour éventuellement cultiver les fourrages. Il faut en effet nourrir la main d'œuvre et l'alimentation humaine a un impact environnemental qu'il s'agira d'évaluer. Alors que l'alimentation humaine a un impact négligeable quand on considère des systèmes mécanisés et à haut niveau d'intrants, elle peut devenir un point incontournable quand on s'intéresse à des systèmes d'élevage à faible niveau d'intrants (par exemple, systèmes pastoraux du Ferlo sénégalais) ou à haute intensité de main d'œuvre (par exemple, systèmes agriculture élevage de la zone cotonnière du Burkina Faso). Le caractère mixte des systèmes agricoles des Suds et la multifonctionnalité des activités d'élevage constituent deux autres particularités importantes. En effet, la majorité des systèmes agricoles associent des activités d'élevage, d'agriculture, et de pisciculture toutes fortement imbriquées et interdépendantes $(7,10)$. De même, les troupeaux remplissent de multiples fonctions, telles que la fourniture de viande et de lait pour l'alimentation humaine, la fourniture d'engrais organiques et de force de traction pour la conduite des cultures $(8,12,13,21)$. Ces particularités ou spécificités supposent une attention particulière à l'allocation des impacts entre les différentes activités de l'exploitation et entre les différentes fonctions des troupeaux. L'allocation protéique (18) ou l'expansion du système (5) sont des voies méthodologiques probablement à privilégier. Enfin, face à un accès limité aux ENR, les systèmes d'élevage tropicaux mobilisent fortement les énergies organiques telles que les biomasses végétales sur parcours et les énergies naturelles telles que le rayonnement solaire (d'ailleurs mobilisées pour la production d'énergies organiques). «Emergy » est une méthode qui justement comptabilise l'utilisation directe et indirecte de ces différentes formes d'énergies et les convertit en une forme d'énergie de référence commune $(4,14,16)$. C'est donc une méthode également intéressante pour évaluer l'inefficience environnementale des systèmes d'élevage dans les Suds et raisonner leur intensification.

\section{- CONCLUSION}

La réflexion autour des bilans énergétiques et GES menée dans cette étude illustre l'intérêt d'une évaluation de l'inefficience environnementale des productions animales tenant compte de l'aval de la production, c'est-à-dire des consommations et émissions directes et indirectes. Au-delà de l'incertitude sur les coefficients énergétiques et les facteurs d'émission, et donc sur les indicateurs environnementaux, ce type d'étude permet de proposer des référentiels et d'identifier des voies de mitigation adaptées. Le cas réunionnais montre que l'inefficience environnementale des systèmes d'élevage est fortement corrélée à leur inefficience zootechnique. Il illustre ainsi qu'il est possible d'intensifier écologiquement les systèmes d'élevage, c'est-à-dire produire plus avec impact environnemental limité.

Les Suds seront les lieux de changements radicaux dans les quarante prochaines années. Or, en agriculture, la majorité des méthodes d'évaluation de type ACV ont été développées en pays du Nord et très peu de références sont aujourd'hui disponibles pour les systèmes d'élevage tropicaux. Un enjeu majeur est l'adaptation de ces méthodes pour leur utilisation en contextes tropicaux. Les premières adaptations méthodologiques ici présentées sur le cas réunionnais illustrent la démarche qui devra être menée et permet d'anticiper des aspects méthodologiques qui seront importants : a) l'adaptation des coefficients énergétiques et des facteurs d'émission, b) la considération en priorité des ressources majeures, parfois originales (par exemple, force de travail humaine et animale), c) l'allocation des impacts dans des systèmes agricoles généralement mixtes et au sein d'activités d'élevage multifonctionnelles (alimentation humaine, fertilisation des cultures, travaux agricoles), et d) une comptabilisation de l'utilisation des autres formes d'énergies (énergies animale, organique et naturelle). 


\section{Remerciements}

Les auteurs remercient les 195 éleveurs qui ont mis à disposition leurs comptabilités via leurs organismes de gestion (CERFrance et chambre d'agriculture) et les provendiers (Urcoopa et Proval-Sanders) qui ont fourni la composition détaillée des aliments d'élevage commercialisés à la Réunion.

\section{BIBLIOGRAPHIE}

1. BASSET-MENS C., BENOIST A., BESSOU C., TRAN T., PERRET S., VAYSSIERES J., WASSENAAR T., 2010. Is LCA-based eco-labelling reasonable? The issue of tropical food products. In: LCA-food VII, Bari, Italy, 22-24 Sept., $6 \mathrm{p}$.

2. BENAGABOU I.N., 2011. Contribution de I'association agricultureélevage dans I'amélioration du bilan du flux énergétique dans les systèmes agro-pastoraux : cas de Koumbia. Mém. Ing. Dév. rural, Université polytechnique, Bobo-Dioulasso, Burkina Faso, 59 p.

3. BOCHU J.L., 2007. Synthèse 2006 des bilans Planète. Consommations d'énergie et émissions de GES des exploitations agricoles ayant réalisé un bilan Planète. Toulouse, France, Solagro/Ademe, 28 p.

4. CASTELLINI C., BASTIANONI S., GRANAI C., DAL BOSCO A., BRUNETTI M., 2006. Sustainability of poultry production using the emergy approach: Comparison of conventional and organic rearing systems. Agric. Ecosyst. Environ., 114: 343-350.

5. CEDERBERG C., STADIG M., 2003. System expansion and allocation in life cycle assessment of milk and beef production. Int. J. Life Cycle Assess., 8: 350-356.

6. CHAUMET J.M., DELPEUCH F., DORIN B., GHERSI G., HUBERT B., LE COTTY T., PAILLARD S., PETIT M., RASTOIN J.L., RONZON T., TREYER S., 2009. Agrimonde : agricultures et alimentations du monde en 2050 : scénarios et défis pour un développement durable. Note de synthèse. Montpellier, France, Cirad/INRA, $195 \mathrm{p}$.

7. D'AQUINO P., LHOSTE P., LE MASSON A., 1995. Interactions entre les systèmes de production, d'élevage et l'environnement : système de productions mixtes agriculture pluviale et élevage en zone humide et sub-humide d'Afrique. Montpellier, France, Cirad-EMVT, $117 \mathrm{p}$.

8. DUGUE P., VALL E., LECOMTE P., KLEIN H.D., ROLLIN D., 2004 Evolution des relations entre l'agriculture et l'élevage dans les savanes

$\stackrel{\sim}{ }$ d'Afrique de l'Ouest et du Centre : un nouveau cadre d'analyse .. pour améliorer les modes d'intervention et favoriser les processus d'innovation. Ol. Corps gras Lipides, 11 : 268-276.

9. GERBER P., VELLINGA T., OPIO C., HENDERSON B., STEINFELD H., 2010. Greenhouse gas emissions from the dairy sector - a life cycle assessment. Rome, Italy, FAO, $94 \mathrm{p}$.

10. HERRERO M., THORNTON P.K., NOTENBAERT A.M., WOOD S., MSANGI S., FREEMAN H.A., BOSSIO D., DIXON J., PETERS M., VAN DE STEEG J., LYNAM J., RAO P.P., MACMILLAN S., GERARD B., MCDERMOTT J., SERE C., ROSEGRANT M., 2010. Smart investments in sustainable food production. Revisiting mixed crop-livestock systems. Science, 327: 822-825.
11. IPCC, 2006. Guidelines for national greenhouse gas inventories. Vol. 4: Agriculture, forestry and other land use. Cambridge, UK, Cambridge University Press, $87 \mathrm{p}$.

12. LANDAIS E., LHOSTE P., 1990. L'association agriculture-élevage en Afrique intertropicale : un mythe techniciste confronté aux réalités du terrain. Cah. Sci. Hum., 26 : 217-235.

13. LANDAIS E., LHOSTE P., 1993. Systèmes d'élevage et transfert de fertilité dans la zone des savanes africaines. II : Les systèmes de gestion de la fumure animale et leur insertion dans les relations entre l'élevage et I'agriculture. Cah. Agric., 2 : 9-25.

14. ODUM H.T., 1984. Energy analysis of the environmental role in agriculture. In: Stanhill G. Ed., Energy and agriculture. Berlin, Germany, Springer Verlag, p. 24-51.

15. RISOUD B., THEOBALD O., 2002. Référentiel pour l'analyse énergétique de l'exploitation agricole et son pouvoir de réchauffement global. Annexe au doc. Analyse énergétique d'exploitations agricoles et pouvoir de réchauffement global. Méthode et résultats sur 140 fermes françaises. Dijon, France, Enesad, Ademe, 43 p.

16. ROTOLO G.C., RYDBERG T., LIEBLEIN G., FRANCIS C., 2007. Emergy evaluation of grazing cattle in Argentina's Pampas. Agric. Ecosyst. Environ., 119: 383-395.

17. STEINFELD H., GERBER P., WASSENAAR T., CASTEL V., ROSALES, M., DE HAAN C., 2006. Livestock's long shadow. Environmental issues and options. Rome, Italy, FAO, LEAD initiative, 319 p.

18. THEVENOT A., VAYSSIERES J., AUBIN J., TILLARD E., 2012. Nitrogen content allocation to handle co-products in livestock systems: Case study on a poultry supply chain. In: LCA-Food VIII, St. Malo, France, 2-4 Oct., 1 p.

19. THEVENOT A., VIGNE M., VAYSSIERES J., 2011. Référentiel pour I'analyse énergétique et I'analyse du pouvoir de réchauffement global des exploitations d'élevage à la Réunion, rapport technique. St Pierre, la Réunion, Cirad, FRCA, 32 p.

20. THORNTON P.K., VAN DE STEEG J., NOTENBAERT A., HERRERO M., 2009. The impacts of climate change on livestock and livestock systems in developing countries: a review of what we know and what we need to know. Agri Syst., 101: 113-127.

21. VALL E., DONGMO NGOUTSOP A.L., NDAO T., ILBOUDO I., 2004. Evolution des pratiques de traction animale et conséquences sur la durabilité des systèmes de culture. Rev. Elev. Méd. vét. Pays trop., 57 : 145-155.

22. VAYSSIERES J., VIGNE M., SLEGTEN V., LECOMTE P., 2009. A whole farm simulation model to improve multi-criteria system efficiency. In: AgSAP int. Conf., Egmond aan Zee, Netherlands, 10-12 Mar. 2009, p. 280-281.

23. VIGNE M., BA A., DEMBELE B., COUliBALY D., 2012. Efficience énergétique des systèmes en intégration agriculture-élevage situés dans la région de Sikasso, Mali Sud. Cah. Econ. rurale, submitted.

Mis en ligne en juin 2013 


\section{Summary}

Vayssières J., Thevenot A., Vigne M., Cano M., Broc A., Bellino R., Diacono E., De Laburthe B., Bochu J.L., Tillard E., Lecomte P. Evaluating zootechnical and environmental inefficiency for ecological intensification of tropical livestock systems. Case study of Reunion Island

According to FAO, animal production would contribute up to $18 \%$ to global anthropogenic green house gas (GHG) emissions. In the face of an increasing world population and demand in food products, more productive and more environmentally-friendly livestock systems have to be conceived. With that aim, non-renewable energy uses and GHG emissions of main animal productions (dairy cattle, beef cattle, pig, poultry and rabbit) were assessed in Reunion tropical island. Based on a method developed in mainland France, energy coefficients and emission factors were redefined to include specificities of the local context and livestock systems. The studied sample comprised 195 farms, i.e. more than $25 \%$ of farms overseen by local cooperatives. The study highlights the positive correlation between environmental inefficiency (non renewable energy uses, and GHG emissions per kilogram of animal product) and zootechnical inefficiency (quantity of concentrate feed consumed per kilogram of animal product). It is thus possible to intensify ecologically animal productions. Similar studies are rare in countries of the South. Their development supposes even more drastic methodological adaptations than those conducted in Reunion so as to evaluate little-mechanized low-input mixed systems, where livestock activities are multifunctional and use various energy types.

Keywords: Animal production - Life cycle analysis - Intensification - Sustainability - Environmental impact - Reunion.

\section{Resumen}

Vayssières J., Thevenot A., Vigne M., Cano M., Broc A., Bellino R., Diacono E., De Laburthe B., Bochu J.L., Tillard E., Lecomte P. Evaluación de las ineficiencias zootécnicas y de ambientales para intensificar ecológicamente los sistemas de cría tropicales. Estudio de un caso en La Reunión

Según la FAO, la cría contribuiría con $18 \%$ de las emisiones globales de gas a efecto de invernadero (GES) de origen antropogénico. Frente a una población mundial y una demanda de productos animales en aumento, se tratan de concebir sistemas de cría no solamente más productivos, sino igualmente más respetuosos del ambiente. Con esta perspectiva, se evaluaron los consumos de energías no renovables (ENR) y las emisiones de GES de las principales producciones animales de La Reunión (bovino de leche, bovino de carne, cerdo, aves y conejo). Partiendo de un método desarrollado en Francia metropolitana, se trató de re evaluar los coeficientes energéticos y los factores de emisión, teniendo en cuenta las particularidades del contexto y de los sistemas de cría locales. La muestra estudiada comprendió 195 establecimientos, o sea más de $25 \%$ de las explotaciones enmarcadas por las cooperativas locales. El presente estudio mostró que las ineficiencias ambientales (consumo de ENR y de emisiones de GES por kilogramo de producto animal) y la ineficiencia zootécnica (cantidades de alimentos concentrados consumidos por kilogramo de producto animal) estuvieron correlacionadas positivamente. Es así posible intensificar ecológicamente producciones animales. Estos estudios son raros en los Sures; su auge supone adaptaciones de metodología aún más importantes que las llevadas en el caso de La Reunión, para poder evaluar los sistemas generalmente poco mecanizados, con bajo nivel de insumos, mixtos, cuya cría es multifuncional y moviliza formas de energía múltiples.

Palabras clave: Producción animal - Análisis del ciclo de duración - Intensificación - Sostenibilidad - Impacto ambiental - Reunión. 
